\title{
Hybrid Active-passive Fault-tolerant Control of Nonlinear NCS based on Event-triggered Communication Scheme
}

\author{
Jun WANG ${ }^{1, a}$, Xiaowan YAO ${ }^{1, b}$, Wei $\mathrm{LI}^{1, \mathrm{c}}$ \\ ${ }^{1}$ Department of Automation, College of Electrical and Information Engineering, Lanzhou University \\ of Technology, Lanzhou, 730050, China \\ a email: Wangj31901@163.com, bemail: jessie_francis@yeah.net, cemail: liwei@lut.cn
}

\begin{abstract}
Keywords: Nonlinear NCS (NNCS); Discrete Event-Triggered Communication Scheme (DETCS);
\end{abstract} Hybrid Active-Passive Fault-Tolerant Control; Fault Detection

\begin{abstract}
The hybrid active-passive fault-tolerant control (FTC) problem based on discrete event-triggered communication scheme (DETCS) is studied for nonlinear NCS (NNCS) with time-varying delay and any actuator failure. A T-S fuzzy model is adopted to describe the fault model of NNCS in which the integration of event-triggered condition and actuator failure is realized. Furthermore, by using of the appropriate constructed Lyapunov-Krasovskii function and $H_{\infty}$ control theory, the sufficient condition that ensure the system possess integrality against any possible actuator failures is deduced and the fault detection observer (FDO) is properly designed, respectively. Moreover, the co-design of FDO and active fault-tolerant controller (AFTC) is also presented, so that the system could maintain stability whenever the actuator failure happens and whatever type the failure is belong to. Finally, a numerical example is given to show the effectiveness of the proposed method.
\end{abstract}

\section{Introduction}

Researching about FTC for NCS has appeals to researcher's attention for years and as a result has managed to achieve some significant progresses[1] [2], but there also many biased.

On one hand, the existing researches mainly about linear NCS, however, for many realistic systems, the controlled plants tend to nonlinear characteristics frequently. At present, researching about FTC for NNCS is still in primary stage [3] [4], waiting for further studying.

On the other hand, the existing FTC methods, mainly divided as active FTC (AFTC) and passive FTC (PFTC), have disadvantages that couldn't be neglected. In spite of this, a new idea named hybrid active-passive fault-tolerant control, which combined the merits of AFTC and PFTC and consequently improved the system performance significantly, has not been widely researched, or in other words, almost none literature referring hybrid active-passive fault-tolerant control for NNCS can be found so far.

Besides this, making a general survey of the achieved results about FTC for NCS, many of which adopt periodic time-triggered communication scheme (PTTCS) as the transmitting scheme, in which data is transmitted with an equal period driven by the physical clock. Considering of the limited network resources, some scholars have recently presented DETCS [5], in which whether or not the sampled state should be transmitted is determined by the current-sampled state and the error between the current-sampled state and the latest transmitted state. Recently, a few scholars introduce the DETCS into fault-tolerant control field for NCS, soon after it is proved that this work is very interesting and valuable in [6] [7].

Above all, this paper mainly researched hybrid active-passive fault-tolerant control for NNCS with time-varying delay and any actuator failure under DETCS, hoping system realize the following functions: when known fault happens, the PFTC that already has been designed off-line, would ensure the system remain stable; whereas when unknown failure occurs, the PFTC would slow down the rate of performance deterioration at the initial period, meanwhile, FDO would estimate the value of fault as soon as possible, then reconstructing controller according to the estimated value to compensate the influence of actuator failure to the system. Consequently, the system would 
always remain stability whenever the failure happens and whatever type the failure is belong to.

\section{Preliminaries and system description}

The introduction of DETCS. The structure of the hybrid active-passive fault-tolerant control of NNCS under DETCS is shown in Figure 1.

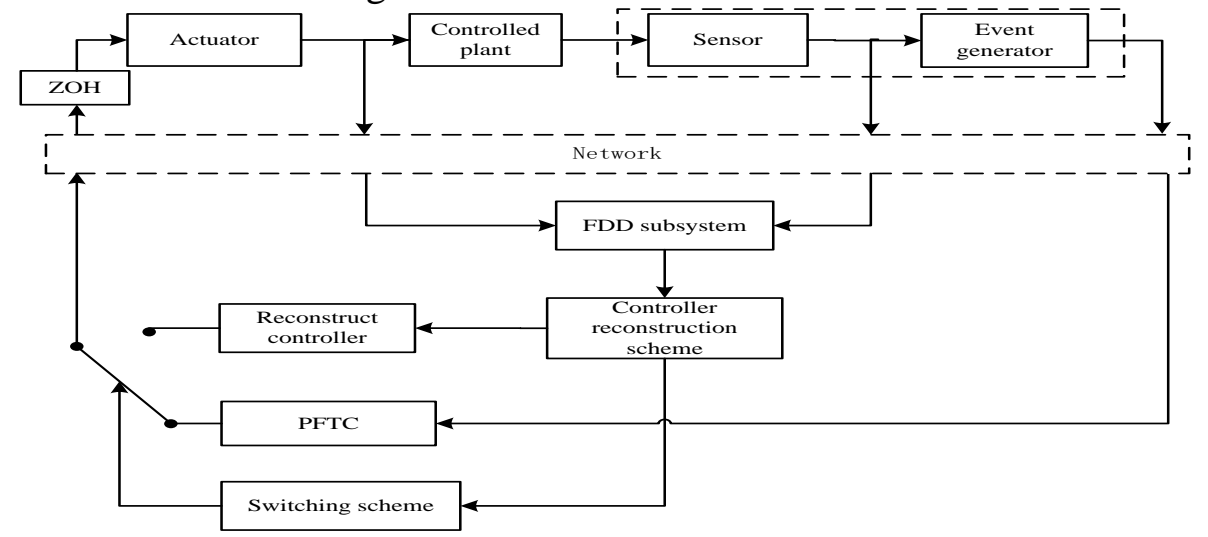

Fig. 1 Structure of the system

For case of convenient exposition, we made the following assumptions.

Assumption1. Sensors are clock-driven. The system states are sampled at a constant period $h, i_{k} h$ is represented for sampled instants, and finally the set of sampled instants is represented by $\left\{i_{k} h \mid i_{k} \in N\right\}$.

Assumption2. Controllers and actuators are event-driven. The transmitted instants $t_{k} h$ are determined by the sampled states $x\left(i_{k} h\right)$. The set of transmission instants is represented by $\left\{t_{k} h \mid t_{k} \in N\right\}$, which is of course a subset of $\left\{i_{k} h \mid i_{k} \in N\right\}$.

Assumption3. The system state is completely measured and it adopts the state-feedback control strategy. Networked-induced delays from sensor to controller $\tau_{t_{k}}^{s c}$, from controller to actuator $\tau_{t_{k}}^{c a}$ and the computational delays $\tau_{t_{k}}^{c}$ are lumped together as $\tau_{t_{k}}=\tau_{t_{k}}^{s c}+\tau_{t_{k}}^{c a}+\tau_{t_{k}}^{c}$.

Assumption4.The detection of fault information is beyond the limitation of the event-triggered condition, that is to say, fault information can be transmitted to fault detection observer at every sampled instants.

Assumption5.The role of the $\mathrm{ZOH}$ is to store the latest data packet, in other words, the actuator keeps the control input unchanged until the output of the $\mathrm{ZOH}$ being updated to a new value.

In contrast to traditional NCS, the sample data needs to pass the event generator before being transmitted by the network, as depicted in Figure 1. The function of the event generator is to determine whether or not to transmit the latest sample signal to controller. The event-triggered condition is presented as following [5]

$$
t_{k+1} h=t_{k} h+\min \left\{l h \mid e_{x}^{T}\left(i_{k} h\right) \Xi e_{x}\left(i_{k} h\right) \geq \sigma x^{T}\left(i_{k} h\right) \Xi x\left(i_{k} h\right)\right\} .
$$

$$
\text { Where } e_{x}\left(i_{k} h\right)=x\left(i_{k} h\right)-x\left(t_{k} h\right) \text {. }
$$

$h$ is the sampling period, $\Xi$ is symmetric positive definite matrix and $\sigma$ is the bounded positive scalar. Only when $x\left(i_{k} h\right)$ and $x\left(t_{k} h\right)$ satisfied event-triggered condition (1), will the event generator be triggered and the sampled data $x\left(i_{k} h\right)$ be transmitted.

The description of NNCS model. Consider a T-S fuzzy system where ith rule of the system is expressed as following

$$
R^{i}: \text { If } \theta_{1}(t) \text { is } N_{i 1} \text { and } \ldots \theta_{g}(t) \text { is } N_{i g} \text {, then }\left\{\begin{array}{l}
\dot{x}(t)=A_{i} x(t)+B_{i} u(t)+E_{i} f(t), i=1,2, \ldots \ldots, r \\
y(t)=C_{i} x(t) .
\end{array}\right.
$$


Where $r$ is the number of rules; $x(t) \in R^{n}$ is the state vector; $u(t) \in R^{m}$ and $y(t) \in R^{p}$ represent input and output vector of the system, respectively; $N_{i s}(s=1,2, \ldots g)$ is the fuzzy set; $\theta(t)=\left[\theta_{1}(t), \theta_{2}(t), \ldots, \theta_{g}(t)\right]^{T}$ denotes the premise variables and assume that it is not rely on $u(t)$; and $A_{i}$, $B_{i}, C_{i}, E_{i}(i=1,2, \ldots, r)$ are constant matrices with appropriate dimensions.

The following T-S fuzzy system state equation can be inferred by using center-average defuzzifier, product inference and a singleton fuzzifier

$$
\left\{\begin{array}{l}
\dot{x}(t)=\sum_{i=1}^{r} h_{i}(\theta(t))\left(A_{i} x(t)+B_{i} u(t)+E_{i} f(t)\right) \\
y(t)=\sum_{i=1}^{r} h_{i}(\theta(t)) C_{i} x(t) .
\end{array}\right.
$$

Where $h_{i}(\theta(t))=\mu_{i}(\theta(t)) / \sum_{i=1}^{r} \mu_{i}(\theta(t)), \mu_{i}(\theta(t))=\prod_{s=1}^{g} N_{i s}\left(\theta_{s}(t)\right)$, and $N_{i s}\left(\theta_{s}(t)\right)$ is the membership value of $\theta_{s}(t)$ in $N_{i s}$. Suppose $\mu_{i}(\theta(t)) \geq 0,(i=1,2, \ldots, r)$ and $\sum_{i=1}^{r} \mu_{i}(\theta(t))>0$, then $h_{i}(\theta(t)) \geq 0$ as well as $\sum_{i=1}^{r} h_{i}(\theta(t))=1$.

NNCS fault model based on DETCS. Based on the previous description of the controlled plant (3), the ith rule of the state-feedback control can be described as

$R^{i}$ : If $\theta_{1}(t)$ is $N_{i 1}$ and $\ldots \theta_{g}(t)$ is $N_{i g}$, then $u(t)=K_{i} x\left(t_{k} h\right), t \in\left[t_{k} h+\tau_{t_{k}}, t_{k+1} h+\tau_{t_{k+1}}\right)$.

Where $K_{i}(i=1,2, \ldots r)$ represent controller gain matrices to be determined in the following theorem, $\tau_{t_{k}}$ and $\tau_{t_{k+1}}$ are network-induced delay at the transmitting instants $t_{k} h$ and $t_{k+1} h$, respectively.

Meanwhile, considering the role of ZOH, when $t \in\left[t_{k} h+\tau_{t_{k}}, t_{k+1} h+\tau_{t_{k+1}}\right)$, the state-feedback control law according to PDC algorithm is shown as following

$$
u(t)=\sum_{i=1}^{r} h_{i}(\theta(t)) K_{i} x\left(t_{k} h\right)
$$

Define $\tau(t)=t-i_{k} h$.

Obviously, $\tau(t)$ is a continuous linear function which meets the requirement of $0<\tau_{m} \leq \tau(t) \leq \tau_{M}$, where $\tau_{m}$ and $\tau_{M}$ are the lower and upper bound of networked-induced delay, respectively.

Combined (2), (4) and (5), $u(t)$ finally can be expressed as following

$$
u(t)=\sum_{i=1}^{r} h_{i}(\theta(t)) K_{i}\left(x(t-\tau(t))-e_{x}\left(i_{k} h\right)\right) .
$$

Supposing fault distribution matrix $E_{i}=-B_{i}, i=1,2, \ldots, r$, and we could define that $L u(t)=u(t)-f(t)$, where $f(t)$ represents actuator failures and unknown matrix $L=\operatorname{diag}\left\{l_{1}, \cdots l_{m}\right\}$, $l_{q} \in[0,1], q=1,2, \cdots, m$ describes fault extent of system actuators, that is, $l_{q}=0$ indicates that the $q$ th actuator is totally invalid; $l_{q}=1$ indicates that the $q$ th actuator operates properly; $l_{q} \in(0,1)$ indicates that the qth actuator is at fault to some extent.

Then the fault model of NNCS can be transformed from (3) to (7) and (8)

$$
\left\{\begin{array}{l}
\dot{x}(t)=\sum_{i=1}^{r} h_{i}(\theta(t))\left(A_{i} x(t)+B_{i} u(t)-B_{i} f(t)\right) \\
y(t)=\sum_{i=1}^{r} h_{i}(\theta(t)) C_{i} x(t) .
\end{array}\right.
$$




$$
\left\{\begin{array}{l}
\dot{x}(t)=\sum_{i=1}^{r} h_{i}(\theta(t))\left(A_{i} x(t)+B_{i} L u(t)\right) \\
y(t)=\sum_{i=1}^{r} h_{i}(\theta(t)) C_{i} x(t)
\end{array}\right.
$$

Remark 1.Obviously, the NNCS fault model (7) is equivalent to (8). The model integrates event-triggered condition, network-induced delay, actuator failures and control law into a unified framework, which lays a solid foundation for the following design of hybrid active-passive fault-tolerant controller for NNCS.

Related lemma. Lemma1.For given symmetric matrix $Z=\left[\begin{array}{ll}Z_{11} & Z_{12} \\ Z_{12}^{T} & Z_{22}\end{array}\right]$, the following three conditions are equivalent

1) $Z<0$;

2) $Z_{11}<0, Z_{22}-Z_{12}^{T} Z_{11}^{-1} Z_{12}<0$;

3) $Z_{22}<0, Z_{11}-Z_{12} Z_{22}^{-1} Z_{12}^{T}<0$.

Lemma2.For any positive definite symmetric matrix $W \in R^{n \times n}, W=W^{T}>0$, scalar $0 \leq h(t) \leq h_{M}$ and vector function $\dot{x}:\left[-h_{M} 0\right] \rightarrow R^{n}$, such that the following integration is well defined

$$
-h(t) \int_{-h(t)}^{0} \dot{x}^{T}(t+s) W \dot{x}(t+s) d s \leq\left[\begin{array}{ll}
x^{T}(t) & x^{T}(t-h(t))
\end{array}\right]\left[\begin{array}{cc}
-W & W \\
W & -W
\end{array}\right]\left[\begin{array}{c}
x(t) \\
x(t-h(t))
\end{array}\right] .
$$

Lemma3 [8].Let $f_{1}, f_{2}, \cdots, f_{N}: R^{m} \rightarrow R$ have positive values in an open subset $D$ of $R^{m}$. Then, the reciprocally convex combination of $f_{i}$ over $D$ satisfies

$$
\min _{\left\{\alpha_{i} \mid \alpha_{i}>0, \Sigma_{i} \alpha_{i}=1\right\}} \sum_{i} \frac{1}{\alpha_{i}} f_{i}(t)=\sum_{i} f_{i}(t)+\max _{g_{i, j}(t)} \sum_{i \neq j} g_{i, j}(t) .
$$

Subject to

$$
\left\{g_{i, j}: R^{m} \rightarrow R, g_{j, i}(t)=g_{i, j}(t),\left[\begin{array}{cc}
f_{i}(t) & g_{i, j}(t) \\
g_{i, j}(t) & f_{j}(t)
\end{array}\right] \geq 0\right\} .
$$

\section{Main Results}

Design of fault detection observer. Considering system model for NNCS (3), if there is no actuator failure, the state of the system can be observed. Supposing $\hat{x}(t) \in R^{n}$ is the estimated state of $x(t), \hat{y}(t)$ is the output of the observer, according to PDC algorithm and NNCS model (7), fault detection observer is designed as following

$$
\left\{\begin{array}{l}
\dot{\hat{x}}(t)=\sum_{i=1}^{r} h_{i}(\theta(t))\left(A_{i} \hat{x}(t)+B_{i} u(t)+G_{i}(y(t-\tau(t))-\hat{y}(t-\tau(t)))\right) \\
\hat{y}(t)=\sum_{i=1}^{r} h_{i}(\theta(t)) C_{i} \hat{x}(t) .
\end{array}\right.
$$

Where $G_{i}$ is gain matrix of the FDO.

Residual, state estimate error and residual error are defined as following, respectively.

$$
\begin{aligned}
& r(t)=W(y(t)-\hat{y}(t)) . \\
& e(t)=x(t)-\hat{x}(t) . \\
& r_{e}(t)=r(t)-f(t) .
\end{aligned}
$$

Where $W$ is residual gain matrix, and as a result

$$
\begin{aligned}
& r(t)=\sum_{i=1}^{r} h_{i}(\theta(t)) W C_{i} e(t) . \\
& \dot{e}(t)=\sum_{i=1}^{r} \sum_{j=1}^{r} h_{i}(\theta(t)) h_{j}(\theta(t))\left(A_{i} e(t)-G_{i} C_{j} e(t-\tau(t))-B_{i} f(t)\right) .
\end{aligned}
$$




$$
r_{e}(t)=\sum_{i=1}^{r} h_{i}(\theta(t))\left(W C_{i} e(t)-f(t)\right) \text {. }
$$

The method of fault estimation based on observer is derived from $H_{\infty}$ control theory, in other words, observer and residual error of real system satisfied such relationship

$\left\|r_{e}(t)\right\|_{2} \leq \gamma^{2}\|f(t)\|_{2}, f(t) \in L_{2}[0, \infty)$.

Where $\gamma$ is a given constant.

Thus, define $H_{\infty}$ performance index as $J_{1}=\int_{0}^{t}\left(r_{e}^{T}(t) r_{e}(t)-\gamma^{2} f^{T}(t) f(t)\right) d t$.

Theorem 1. For given positive scalar $\tau_{m}, \tau_{M}, \tau_{s}, a, b, c, \varepsilon$, if there exist positive definite symmetric matrices $P, Q_{i}, V_{i}, W$ which satisfied the following Linear Matrix Inequality (LMI)

$$
\Phi=\left[\begin{array}{ccccccccc}
\Phi_{11} & \Phi_{12} & \Phi_{13} & \Phi_{14} & \Phi_{15} & \Phi_{16} & \Phi_{17} & \Phi_{18} & \Phi_{19} \\
* & \Phi_{22} & \Phi_{23} & \Phi_{24} & 0 & 0 & \Phi_{27} & \Phi_{28} & \Phi_{29} \\
* & * & \Phi_{33} & \Phi_{34} & 0 & 0 & 0 & 0 & 0 \\
* & * & * & \Phi_{44} & 0 & 0 & 0 & 0 & 0 \\
* & * & * & * & \Phi_{55} & \Phi_{56} & \Phi_{57} & \Phi_{58} & \Phi_{59} \\
* & * & * & * & * & \Phi_{66} & 0 & 0 & 0 \\
* & * & * & * & * & * & \Phi_{77} & 0 & 0 \\
* & * & * & * & * & * & * & \Phi_{88} & 0 \\
* & * & * & * & * & * & * & * & \Phi_{99}
\end{array}\right]<0 .
$$

Where * represents the corresponding matrix to be obtained through the symmetric matrix.

$\Phi_{11}=P A_{i}+A_{i}^{T} P+Q_{1}-Q_{3}-Q_{4}, \Phi_{12}=-V_{i} C_{j}, \Phi_{13}=Q_{3}, \Phi_{14}=Q_{4}, \Phi_{15}=-P B_{i}, \Phi_{16}=C_{j}^{T} W^{T}$,

$\Phi_{17}=\tau_{m} A_{i}^{T} P, \Phi_{18}=\tau_{M} A_{i}^{T} P, \Phi_{19}=\tau_{s} A_{i}^{T} P, \Phi_{22}=-2 Q_{5}+M_{12}^{T}+M_{12}, \Phi_{23}=Q_{5}-M_{12}, \Phi_{24}=Q_{5}-M_{12}^{T}$,

$\Phi_{27}=-\tau_{m} C_{j}^{T} V_{i}^{T}, \Phi_{28}=-\tau_{M} C_{j}^{T} V_{i}^{T}, \Phi_{29}=-\tau_{s} C_{j}^{T} V_{i}^{T}, \Phi_{33}=Q_{2}-Q_{1}-Q_{3}-Q_{5}, \Phi_{34}=M_{12}^{T}$,

$\Phi_{44}=-Q_{2}-Q_{4}-Q_{5}, \Phi_{55}=-\gamma^{2} I, \Phi_{56}=-I, \Phi_{57}=-\tau_{m} B_{i}^{T} P, \Phi_{58}=-\tau_{M} B_{i}^{T} P, \Phi_{59}=-\tau_{s} B_{i}^{T} P$,

$\Phi_{66}=-I, \Phi_{77}=-2 a P+a^{2} Q_{3}, \Phi_{88}=-2 b P+b^{2} Q_{4}, \Phi_{99}=-2 c P+c^{2} Q_{5}$.

Then we can obtain observer gain matrix through formula $G_{i}=P^{-1} V_{i}$ and the fault detection observer could ensure the fault estimation error meets the requirement of $\left\|r_{e}(t)\right\|_{2} \leq \gamma^{2}\|f(t)\|_{2}$.

The proof to theorem 1 is omitted, if there is a need for it, please contact the author.

Design of PFTC. Aimed at system fault model (8), PFTC is designed as following.

According to formula (6), the state-feedback controller could be written as

$$
u_{p}(t)=\sum_{i=1}^{r} h_{i}(\theta(t)) K_{p i}\left(x(t-\tau(t))-e_{x}\left(i_{k} h\right)\right) \text {. }
$$

Where $K_{p i}$ represents gain matrix of the PFTC, which ensure the system keeps asymptotically stable in the event of any possible actuator failure.

Theorem 2. Under the event-triggered condition (1) in the DETCS, for the given constants $\sigma \in[0,1), \tau_{m}, \tau_{M}, \tau_{s}$, if there exist some positive definite symmetric matrices $R_{i}>0, i=1,2, \ldots 5$ and $V, Y_{j}$, for any possible actuator failures in mode set $L$, these parameters satisfy the following LMI 


$$
\Phi=\left[\begin{array}{cccccccc}
\Phi_{11} & \Phi_{12} & \Phi_{13} & \Phi_{14} & \Phi_{15} & \Phi_{16} & \Phi_{17} & \Phi_{18} \\
* & \Phi_{22} & \Phi_{23} & \Phi_{24} & 0 & \Phi_{26} & \Phi_{27} & \Phi_{28} \\
* & * & \Phi_{33} & \Phi_{34} & 0 & 0 & 0 & 0 \\
* & * & * & \Phi_{44} & 0 & 0 & 0 & 0 \\
* & * & * & * & \Phi_{55} & \Phi_{56} & \Phi_{57} & \Phi_{58} \\
* & * & * & * & * & \Phi_{66} & 0 & 0 \\
* & * & * & * & * & * & \Phi_{77} & 0 \\
* & * & * & * & * & * & * & \Phi_{88}
\end{array}\right]<0 .
$$

Where $*$ represents the corresponding matrix to be obtained through the symmetric matrix.

$$
\begin{aligned}
& \Phi_{11}=A_{i} X+X A_{i}^{T}-2 X-R_{1}+R_{3}+R_{4}, \Phi_{12}=B_{i} L Y_{j}, \Phi_{13}=2 X-R_{3}, \Phi_{14}=2 X-R_{4}, \Phi_{15}=-B_{i} L Y_{j}, \\
& \Phi_{16}=\tau_{m} X A_{i}^{T}, \Phi_{17}=\tau_{M} X A_{i}^{T}, \Phi_{18}=\tau_{s} X A_{i}^{T}, \Phi_{22}=\sigma(2 X-V)+2 R_{5}-R_{6}-R_{6}^{T}, \Phi_{23}=R_{6}-R_{5}, \\
& \Phi_{24}=R_{6}^{T}-R_{5}, \Phi_{26}=\tau_{m} Y_{j}^{T} L B_{i}^{T}, \Phi_{27}=\tau_{M} Y_{j}^{T} L B_{i}^{T}, \Phi_{28}=\tau_{s} Y_{j}^{T} L B_{i}^{T}, \Phi_{33}=R_{1}+R_{3}+R_{5}-R_{2}-4 X, \\
& \Phi_{34}=2 X-R_{6}^{T}, \Phi_{44}=R_{2}+R_{4}+R_{5}-6 X, \Phi_{55}=-2 X+V, \Phi_{56}=-\tau_{m} Y_{j}^{T} L B_{i}^{T}, \Phi_{57}=-\tau_{M} Y_{j}^{T} L B_{i}^{T}, \\
& \Phi_{58}=-\tau_{s} Y_{j}^{T} L B_{i}^{T}, \Phi_{66}=-R_{3}, \Phi_{77}=-R_{4}, \Phi_{88}=-R_{5} .
\end{aligned}
$$

Then there exist state-feedback control law (6) which keeps the NNCS fault model (8) asymptotically stable. That is, (6) denotes the state-feedback control law with which the NNCS fault model (8) possesses robust integrity, where controller gain matrix and event-triggered weight matrix could be computed through $K_{j}=Y_{j} X^{-1}$ and $\Xi=V^{-1}$, respectively.

The proof to theorem 2 is omitted, if there is a need for it, please contact the author.

Design of hybrid fault-tolerant controller. Assuming $\operatorname{rank}\left(F, E_{i}\right)=\operatorname{rank}(F)$ is true firstly, according to theorem from literature [7], under the circumstance of the previous assumption, there exists matrix $F^{+} \in R^{m \times n}$ makes the following equality $\left(I-F F^{+}\right) E_{i}=0$ true.

Set hybrid fault-tolerant compensation controller based on fault detection observer is

$$
u(t)=u_{p}(t)-F^{+} E_{i} \hat{f}(t) \text {. }
$$

Where $F^{+}$is the right false inverse matrix of $B_{i}, u_{p}(t)$ is the designed PFTC from theorem 2, $\hat{f}(t)$ represents the estimate value of failure.

$$
\begin{aligned}
& \text { Applying (16) to (7), we obtain } \\
& \qquad \begin{aligned}
\dot{x}(t) & =\sum_{i=1}^{r} h_{i}(\theta(t))\left[A_{i} x(t)+B_{i}\left(u_{p}(t)-F^{+} E_{i} \hat{f}(t)\right)+E_{i} f(t)\right] \\
& =\sum_{i=1}^{r} h_{i}(\theta(t))\left[A_{i} x(t)+B_{i} u_{p}(t)-E_{i}(\hat{f}(t)-f(t))\right] \\
& =\sum_{i=1}^{r} h_{i}(\theta(t))\left[A_{i} x(t)+B_{i} u_{p}(t)-E_{i} e_{f}(t)\right] \\
y(t) & =\sum_{i=1}^{r} h_{i}(\theta(t)) C_{i} x(t)
\end{aligned}
\end{aligned}
$$

Where $e_{f}(t)$ represents fault estimate error.

In consideration of fault detection observer designed in theorem 1 which satisfied the following performance index $\left\|r_{e}(t)\right\|_{2} \leq \gamma^{2}\|f(t)\|_{2}, f(t) \in L_{2}[0, \infty)$ and according to theorem 2, $u_{p}(t)$ could ensure the system (8) asymptotically stable, so we can get the conclusion that the formula (17) is asymptotically stable as well.

\section{Simulation Experiment and Result Analysis}

Adopt NNCS model data in literature [8] and the fuzzy membership function as $M_{1}\left(x_{2}\right)=\sin ^{2} x_{2}$ 
and $M_{2}\left(x_{2}\right)=\cos ^{2} x_{2}$, then the system model could be expressed as the following T-S fuzzy system of two rules.

$$
\begin{aligned}
& R^{1}: \text { If } x_{2} \text { is } M_{1} \text {, then }\left\{\begin{array}{l}
\dot{x}(t)=A_{1} x(t)+B_{1} u(t)+E_{1} f(t) \\
y(t)=C_{1} x(t)
\end{array}\right. \\
& R^{2}: \text { If } x_{2} \text { is } M_{2} \text {, then }\left\{\begin{array}{l}
\dot{x}(t)=A_{2} x(t)+B_{2} u(t)+E_{2} f(t) \\
y(t)=C_{2} x(t)
\end{array} .\right.
\end{aligned}
$$

Where $A_{1}=\left[\begin{array}{cc}-3 & 1 \\ 1 & -1\end{array}\right], A_{2}=\left[\begin{array}{cc}-2 & 1 \\ 1 & 0\end{array}\right], B_{1}=\left[\begin{array}{cc}1 & 0 \\ 0 & -0.5\end{array}\right], B_{2}=\left[\begin{array}{cc}1 & 0 \\ 0 & 0.5\end{array}\right], C_{1}=\left[\begin{array}{cc}1 & 0 \\ 0 & 1\end{array}\right], C_{2}=\left[\begin{array}{cc}1 & 0 \\ 0 & -1\end{array}\right]$.

Setting parameters as following $h=0.05 s, \tau_{m}=0.2, \tau_{M}=0.3, \tau_{s}=0.1, \sigma=0.3, a=0.1, b=0.2$, $c=0.5$.

For actuator normal or fault, matrix $L$ is defined as following:

$$
L_{0}=\left[\begin{array}{ll}
1 & 0 \\
0 & 1
\end{array}\right], \quad L_{1}=\left[\begin{array}{cc}
0.2 & 0 \\
0 & 0.8
\end{array}\right], \quad L_{2}=\left[\begin{array}{ll}
1 & 0 \\
0 & 0
\end{array}\right] \text {. }
$$

According to theorem 2, we obtain the state-feedback controller gain matrices and the event-triggered weight matrix as following:

$$
K_{1}=\left[\begin{array}{cc}
-0.1666 & -0.0602 \\
-0.1996 & 0.5762
\end{array}\right], \quad K_{2}=\left[\begin{array}{cc}
-0.1922 & -0.2265 \\
-0.2978 & -0.2203
\end{array}\right], \quad \Xi=\left[\begin{array}{cc}
0.0033 & -0.0009 \\
-0.0009 & 0.0016
\end{array}\right] .
$$

Similarly, according to theorem 1, we obtain the fault detection observer gain matrices and the residual gain matrix as following:

$$
G_{1}=\left[\begin{array}{cc}
-0.3506 & 0.5883 \\
0.4193 & 0.5824
\end{array}\right], G_{2}=\left[\begin{array}{ll}
0.1181 & 0.6818 \\
0.4257 & 0.4437
\end{array}\right], W=\left[\begin{array}{cc}
1.5662 & -0.1369 \\
-0.1369 & -1.6215
\end{array}\right] \text {. }
$$

Assuming the system operates normally before $t=5 \mathrm{~s}$, and known faults begin to appear at the instant $t=5 \mathrm{~s}$, unknown fault such as $f=\left[\begin{array}{c}0 \\ 0.5\end{array}\right]$ begins to appear at the time $t=10 \mathrm{~s}$.

The simulation results are shown as following: The response curve of the estimation of faults is shown in Figure 2.

From Figure 2 we could make out that even though the fault estimated value lag behind fault input value at the instants $t=5 \mathrm{~s}$ and $t=10 \mathrm{~s}$, they are fit closely at all other times, which illustrated the validity of the fault estimation method that we have adopted.

Setting initial condition $x(0)=\left[\begin{array}{ll}2 & -2\end{array}\right]^{T}$, under the aforementioned actuator failures in mode set $L$ and the results contrasting figures of state component $x_{1}, x_{2}$ in passive (S1), active (S2) and hybrid (S3) fault-tolerant control are shown in Figure 3 and 4, respectively.

Observing Figure 3 and 4, we can conclude that, when actuator operates normally, both AFTC and PFTC could keep the system stable; when actuator appears known faults, PFTC could tolerant the fault effectively as well as keeps the system stable, whereas AFTC needs to reconstruct the controller according to the value of detected failure, and this certainly will waste some time to help the system recovery stable; on the contrary, when there happen unknown faults, PFTC lost the ability to tolerant faults and the system become unstable, but AFTC begins to show its superiority, compared to PFTC, AFTC reacts more quickly to failure. Though there appears amplitude jump at the initial period of failure, it executes fault detection and controller reconstruction rapidly and finally the system become stable again; the most significant aspect is that compared to AFTC and PFTC, adopting hybrid fault-tolerant control method not only can keeps the system stable when known failure happens, but also can slow down the rate of the system performance deterioration when unknown fault occurs, meanwhile, reconstructing controller rapidly and finally makes the system recovery stable. Above all, hybrid active-passive fault-tolerant control method combined the merits of AFTC and PFTC, which contribute much to the improving of the system's stability. 


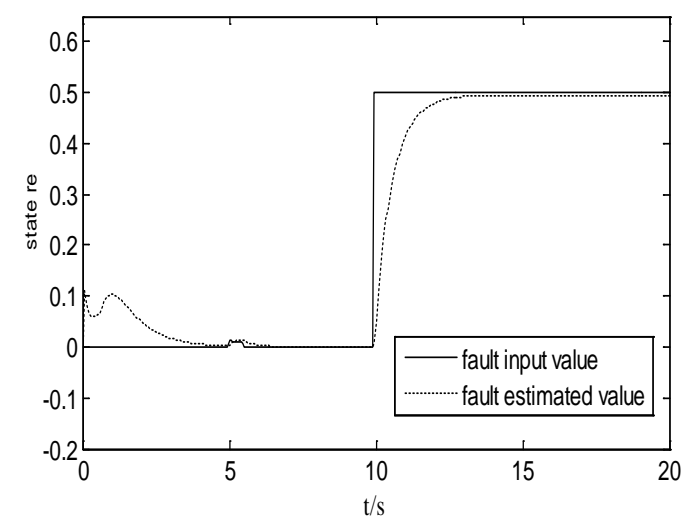

Fig.2 Response curve of the estimation of faults

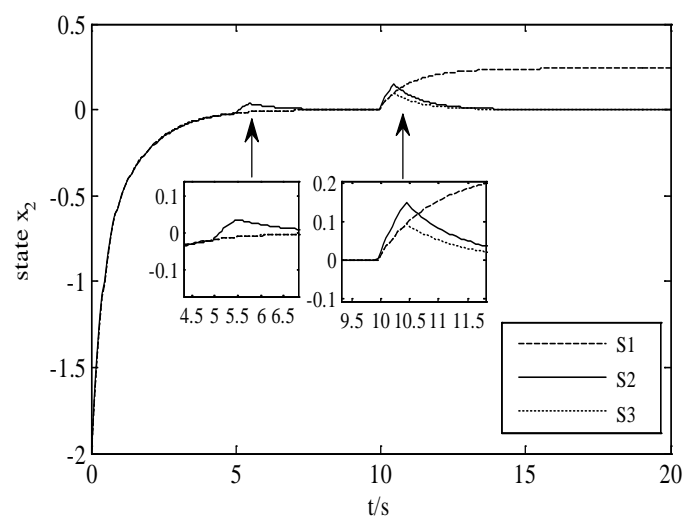

Fig.4 Response curve of state $x_{2}$ in different control action

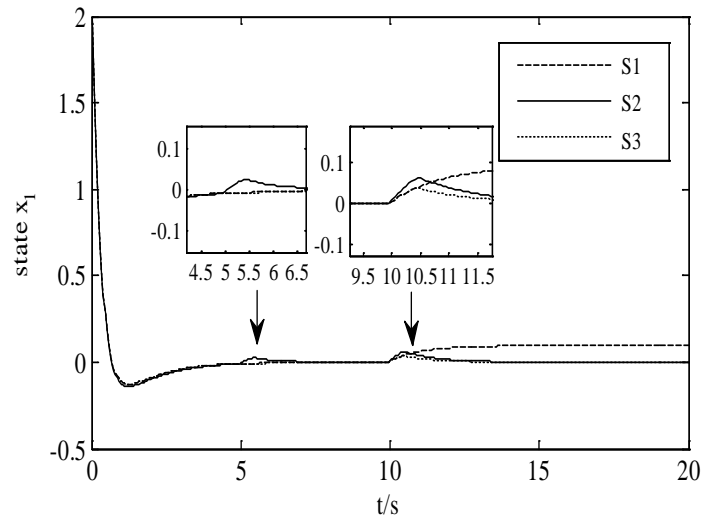

Fig.3 Response curve of state $x_{1}$ in different control action

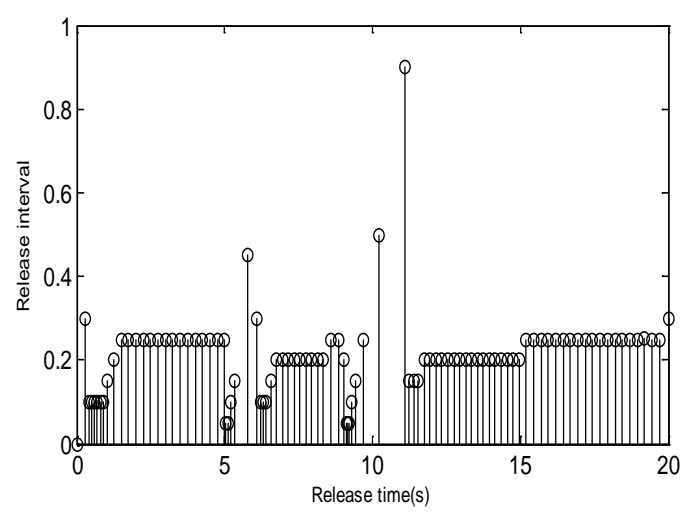

Fig.5 Release time(s) and release interval of data transmission for hybrid active-passive

fault-tolerant control of NNCS based on

DETCS

Similarly, under event-triggered condition (1) in the DETCS and with simulation time $t=20 \mathrm{~s}$, the relationship between release time(s) and release interval of data transmission is shown in Figure 5 as following.

As can be seen in Figure 5, the quantity of data transmission significantly decreases and as a consequence we can conclude that the DETCS driven by control demand is indicated to occupy less network resources than the PTTCS driven by a physical clock.

\section{Conclusions}

This paper mainly researched NNCS with time-varying delay based on DETCS, under the circumstance of reduce network resources, has realized the integrated-design of NNCS faultdetection observer and hybrid active-passive fault-tolerant controller. As has demonstrated in the above chapter, this new method not only can detect failures effectively, whether they are time-varying or not, but also can ensure the system keeps stability whatever type the failure is belong to. Finally, a numerical example is given to show the effectiveness of the proposed method.

\section{Acknowledgement}

In this paper, the research was sponsored by the National Natural Science Foundation of China (Grant No. 61463030, 61563031), Youth Science and Technology Foundation of Gansu Province (148RJYA007) and Open Projects of Key Laboratory of Gansu Advanced Control for Industrial Processes (XJK201521). 


\section{References}

[1] Duan K, Zhang W D. Event-triggered fault-tolerant control for networked systems with dynamic quantiser [J]. Control Theory and Applications. 2016, 10(9): 1088-1096.

[2] PooGeon Park, Jeong Wan Ko, Changki Jeong. Reciprocally convex approach to stability of systems with time-varying delays [J]. Automatica, 2010, 47(1): 235-238.

[3] Wang Y F, Jiang Y W, Zhang S Y. Fault-tolerant control for nonlinear networked control systems based on observer [J]. Control Theory and Applications. 2012, 29(10): 1348-1352.

[4] Jiang D N, Li W. Dynamic output feedback-based robust performance guaranteeing fault-tolerant control of nonlinear networked control system with uncertainty [J]. Journal of Lanzhou University of Technology, 2015, 41(4): 83-89.

[5] W Li, K Yan. The robust integrity design of uncertain NNCS based on discrete event-triggered scheme [J]. Journal of Lanzhou University of Technology, 2015, 41(2): 83-90.

[6] Yong Zhang, Huajing Fang, Zhen Luo. $H_{\infty}$-based fault detection for nonlinear networked systems with random packet dropout and probabilistic interval delay [J]. Journal of Systems Engineering and Electronics, 2011, 22(5): 825-831.

[7] QIU Ai-Bing, JI Hong-Gang, GU Ju-Ping. Optimal Integrated Design of Time-varying Fault Estimation and Accommodation for Non-uniformly Sampled Data Systems [J]. ACTA AUTOMATICA SINICA, 2014, 40(7): 1493-1504.

[8] Wang Jun, Li Wei, Guan Ling. Fuzzy Observer-based Robust $H_{\infty}$ Guaranteed Cost Fault-tolerant Design for Nonlinear NCS [J]. Open Electrical and Electronic Engineering Journal, 2014, 8(1): 71-76 